\section{PENSAR LA COMUNICACIÓN PARA EL CAMBIO SOCIAL EN ESPAÑOL AQUÍ Y AHORA}

\author{
Víctor Manuel Marí Sáez \\ Universidad de Cádiz \\ http://orcid.org/0000-0003-4939-3776 \\ victor.mari@uca.es
}

\section{THINKING COMMUNICATION FOR SOCIAL CHANGE IN SPANISH HERE AND NOW}

Cómo citar este artículo/Citation: Víctor Manuel Marí Sáez (2021). Pensar la Comunicación para el Cambio Social en español aquí y ahora. Arbor, 197(801): a615. https://doi. org/10.3989/arbor.2021.801005

Recibido: 15 febrero 2020. Aceptado: 7 mayo 2021.

Publicado: 9 diciembre 2021

Resumen: En la actualidad asistimos a un renacer del pensamiento comunicacional latinoamericano (Marques de Melo, 2009) en el seno de las ciencias de la comunicación, que propone una mirada reflexiva y alternativa a las lógicas comunicativas del capitalismo global. El texto de Reyes Mate, Pensar en español aquí y ahora (2008), se utiliza en este artículo como piedra angular del análisis de este renacer, por su capacidad para estimular el estudio de las aportaciones específicas de la Comunicación para el Cambio Social en español al conjunto del campo de la comunicación y, quizá, también a otros campos del conocimiento. Así, las ideas que lo estructuran son: 1) el pensa-miento comunicación en español es, en la actualidad, un pensa-miento dependiente; 2) la comunicación no puede ser inmoral y 3) Pensar la comunicación desde el sur para un pensamiento comunicacional crítico de ida y vuelta.

PALABRAS CLAVE: comunicación y cambio social, pensamiento comunicacional, Reyes Mate, capitalismo académico, desarrollo, postdesarrollo.
Copyright: @ 2021 CSIC. Este es un artículo de acceso abierto distribuido bajo los términos de la licencia de uso y distribución Creative Commons Reconocimiento 4.0 Internacional (CC BY 4.0).

Abstract: We are currently witnessing a rebirth of Latin American communicational thought within the Communication Sciences, which proposes a reflective and alternative view of the communicative logics of global capitalism. We take as a cornerstone, for analysis of this phenomenon, the text by Reyes Mate, Pensar en español aquí y ahora (2008), for its ability to stimulate analysis of the specific contributions of Communication for Social Change in Spanish to the entire field of Communication and, perhaps, also to other fields of knowledge. To this end, the present article is organized around the following ideas: 1) Communication thinking in Spanish is, at present, a dependent thought; 2) Farewell to Aristotle: communication cannot be immoral; and 3) Thinking communication from the South: for a round trip of critical communicative thinking.

KEYWORDS: Communication and Social Change; Communicative thinking; Reyes Mate; academic capitalism; development; postdevelopment. 


\section{INTRODUCCIÓN}

Pensar la comunicación. A la ciudadanía contemporánea le puede extrañar esta expresión ya que, lo habitual es practicar la comunicación, más que pensarla. En los tiempos de las redes sociales virtuales, de la constante mediatización de la sociedad (Couldry y Hepp, 2013), de las comunicaciones ininterrumpidas (Marí, 2016a) como horizonte totalizante que lo envuelve todo, parece extraño remitir a una dimensión reflexiva vinculada al acto comunicativo.

Desgraciadamente, este lugar común que lleva a comprender la comunicación como una actividad más práctica que teórica, ha tenido su reflejo en el terreno científico a la hora de configurar el campo de las ciencias de la comunicación. Desde los trabajos clásicos en torno a las teorías de la comunicación de Armand y Michèle Mattelart (1997) hasta aproximaciones más recientes sobre el devenir de la comunicación como campo académico (Waisbord, 2019), existe una tensión esquizofrénica entre quienes ubican la comunicación como un saber instrumental/profesional y los que la conciben como un saber científico. En España, esta tensión también tiene su reflejo en el proceso de institucionalización de las ciencias de la comunicación. Uno de los estudiosos del caso español, Manuel Martínez Nicolás (2006, 2019), aludía precisamente a esta tensión y denominaba "practicistas» a quienes entienden la formación universitaria en materia de comunicación en un sentido estrictamente instrumental, capacitante para el ejercicio profesional en las empresas, y «teoricistas", a quienes creen que las ciencias de la comunicación constituyen un ámbito científico específicamente orientado al análisis de la comunicación y, en general, de la cultura de masas (Martínez Nicolás, 2019: 43). Por último, para acabar de rizar el rizo, el estudio genealógico del campo de la comunicación remite a otra tensión, la que lleva a adscribirlo en las Ciencias Humanas o en las Ciencias Sociales. Las instituciones encargadas de clasificar las áreas de conocimiento y de la investigación han incorporado la comunicación al grupo de las Ciencias Sociales, despreciando su capacidad de ser «interlocutoras del saber humanístico» (Marzal, Rodríguez y Soler, 2019: 110).

En este contexto y, como contrapunto a las tendencias expuestas, las aportaciones teóricas latinoamericanas remiten desde la década de 1970 a la importancia de plantear un «pensamiento comunicacional» (Marques de Melo, 2009) ${ }^{1}$ que contrarreste los excesos procedentes del empirismo y del funcionalismo subyacentes a la mirada del campo comunicativo preferentemente práctica, configurada históricamente en Estados Unidos y en Europa. Una parte importante de la investigación en comunicación que ha cristalizado en el continente latinoamericano, desde ese periodo hasta la actualidad, ha permitido pensar la comunicación en estrecha conexión con los procesos de cambio social y subrayar la orientación marcadamente crítica del pensamiento comunicacional emergente. Así, en la actualidad existe una cierta eclosión de posicionamientos críticos en el seno de las ciencias de la comunicación, especialmente en los subcampos de las denominadas Comunicación para el Desarrollo (Servaes, 2002), Comunicación para el Cambio Social (Gumucio y Tufte, 2006) y Comunicación para el Empoderamiento (Chaparro, 2012), por mencionar solo algunas de las múltiples conceptualizaciones en disputa.

Este reverdecimiento del pensamiento comunicacional de carácter crítico requiere de un análisis fundamentado. Las aportaciones de Reyes Mate en torno al «pensar en español» pueden resultar útiles para esta tarea. Esta expresión, que Mate utilizó como título del artículo que publicó en 2008, le había servido para dar nombre a su aportación en «una serie de coloquios cuyo objetivo era reflexionar sobre los supuestos, contenidos y posibilidades de conformar una comunidad cultural iberoamericana» (Mate, 2008: 979). Posteriormente, utilizó esta idea central, «pensar en español», como punto de partida para su aportación en la monumental y referencial obra de la Enciclopedia Iberoamericana de Filosofía, en cuyo

1 Entre el pensamiento comunicacional latinoamericano y el pensamiento comunicacional en español hay una serie de continuidades y discontinuidades que serán abordadas en el tercer epígrafe de este texto. La primera y más evidente de esas discontinuidades, es la relativa al idioma. Lo latinoamericano incluye, además del español, los idiomas de raíz latina hablados en otros países de Latinoamérica (portugués y francés). Gran parte de los análisis realizados aquí sobre el pensamiento comunicacional en español son también aplicables al francés y al portugués. Entrar a abordar los matices específicos excedería los límites de este texto. Por otro lado, preferimos mantener la expresión «pensamiento comunicacional latinoamericano» porque está ya asentada en la literatura científica sobre el tema. Del mismo modo, mantenemos la expresión «pensar la comunicación en español» porque nos permite salvar la continuidad con las reflexiones del texto de Reyes Mate que ha inspirado las ideas aquí expuestas, aunque él mismo se abre a la extensión de sus reflexiones al portugués. Véase, por ejemplo, la Enciclopedia Iberoamericana de Filosofía, concreción de las reflexiones que están en el sustrato y en los orígenes de su texto, así como al número monográfico publicado en esta revista sobre el Pensar en español (número 734, 2008), con la valiosa contribución al debate de Luis Villoro y de otras personas especialistas en el tema. 
proceso de gestación jugó un papel crucial. Los argumentos de Reyes Mate pueden ser útiles, en un aquí y una ahora diferentes, para analizar las aportaciones específicas que puede plantear la comunicación para el cambio social en español al conjunto del campo de la comunicación y quizá, también, a otros campos del conocimiento. Se destacan a continuación las ideas de Reyes Mate que inspiran este texto, así como, la reelaboración y adaptación que se ha hecho a partir de ellas para responder al objetivo central del mismo:

1. El «pensar en español» es un pensamiento dependiente.

Este diagnóstico es de lo más pertinente en la actualidad cuando se aplica al análisis del campo comunicacional, que está atravesado por múltiples dependencias; entre ellas: 1) la dependencia respecto al capitalismo, que llevan a subsumir a la comunicación en las lógicas de la mercancía y de la razón instrumental (Fuchs, 2020); y 2) la dependencia respecto al pensar en inglés, que predomina en la actualidad en el campo académico y que va más allá de la imposición formal de un idioma. La supremacía del inglés llega a condicionar aspectos tan fundamentales como son los objetos de estudio, las formas de pensamiento y de expresión o los formatos de publicación y de difusión del conocimiento (Ortiz, 2009).

2. Los límites del pensamiento aristotélico: el pensar no puede ser inmoral.

Esta idea surge en el contexto de la conocida disputa entre Bartolomé de las Casas y Ginés de Sepúlveda a propósito de la conquista de América y, al hilo de ella, de la controversia en torno a la consideración del indígena como sujeto de derechos. Reyes Mate retomó el dilema en el que se encontraba el fraile dominico, entre un saber racional, pero injusto, dominante en la época (basado, entre otros, en el pensamiento de Aristóteles) y su experiencia directa sobre las consecuencias negativas de la dominación española para los pueblos originarios del continente americano.

En esa línea argumental planteada por Bartolomé de las Casas y retomada por Reyes Mate, aparece una sugerente analogía entre la toma de distancia que hace de Las Casas respecto a la irracionalidad de la racionalidad occidental dominante y el correspondiente distanciamiento de un grupo importante de investigadoras e investigadores latinoamericanos del campo de la comunicación respecto a las teorías de matriz aristotélica implícitas en las teorías de la comunicación estadounidenses y europeas.

3. Pensar la comunicación desde el Sur: por un pensamiento comunicacional crítico de ida y vuelta.

En el texto de referencia de Reyes Mate, este alude a un pensar desde el Sur que lleve a España y a Portugal a arrastrar a Europa hacia unas corrientes de solidaridad con el Sur, alejadas del universalismo abstracto tan propio del Norte. Esta idea se utiliza en este artículo como anclaje y se conecta con las reflexiones actuales en torno a las epistemologías del Sur (Sousa Santos, 2009) y de una comunicación desoccidentalizada (Waisbord y Mellado, 2014), para explorar sus posibilidades y límites.

Así, las tres ideas centrales tomadas de Reyes Mate (2008), adaptadas al objetivo de este análisis, articulan los tres epígrafes de este artículo: 1) el pensamiento comunicación en español es, en la actualidad, un pensamiento dependiente; 2) Adiós a Aristóteles: la comunicación no puede ser inmoral y 3) Pensar la comunicación desde el Sur: por un pensamiento comunicacional crítico de ida y vuelta.

\section{UN PENSAMIENTO COMUNICACIONAL EN ES- PAÑOL DEPENDIENTE}

Las primeras teorías de la comunicación, gestadas en el seno del funcionalismo en el primer tercio del siglo XX, se caracterizaron por mostrar un fuerte reduccionismo de los elementos contextuales en los que se desenvuelve la comunicación de masas. Las famosas preguntas del modelo de Harold Lasswell (1948) en torno a las que analizar el proceso de comunicación-quién dice qué, a quién, a través de qué canal y con qué efecto -parten de un silencio elocuente sobre el entramado social en el que se inserta la actividad comunicativa ${ }^{2}$. Así, según este modelo, que sigue vigente a pesar de las críticas, la comunicación se presenta como encerrada en una campana

2 Las cinco preguntas que Lasswell propone en su modelo de comunicación se conocen como la regla de las $5 \mathrm{~W}$ pues, cada una de ellas, en el idioma original en que están formuladas, el inglés, incluye una "w" en algún sitio: "Who (comunicator) says what (message) in which chanel (médium) to whom (receiver) with what effect (effect)?». 
de cristal, como un acto técnico ajeno a la contaminación que, desde esta perspectiva, implica el anclaje en lo social. Desde el punto de vista alternativo, parecería obvio que es imposible levantar un pensamiento comunicacional al margen de la contextualización sociopolítica del mismo; pero estas primeras teorías comunicativas descuidaron las preguntas en torno a lo social, sorprendentemente, también a la hora de plantear una alternativa al modelo comunicativo dominante.

La realidad es que muchos de los planteamientos contemporáneos en torno a la comunicación alternativa se construyen desde el descuido, a veces ceguera, respecto a los estrechos márgenes que el capitalismo ofrece a cualquier propuesta que quiera situarse fuera de sus lógicas de dominio sistémico. No se está planteando aquí una resignación frente al imperio de lo dado; se trata más bien de una apelación a un pensamiento que vaya más allá del adanismo del sí se puede, cuando esta afirmación surge de un deseo autoafirmativo e inmediato de transformación social, más propio de la literatura barata de autoayuda que de un análisis social mínimamente consistente. La supuesta alternatividad de la comunicación, cuando no parte de un análisis de sus condiciones de posibilidad en el seno del dominio totalizante del capitalismo, puede retroalimentar los mecanismos de dependencia más que ponerlos en cuestión. Así lo muestran, por ejemplo, dos investigaciones sobre el análisis de la institucionalización de la comunicación para el desarrollo y el cambio social en España, pues reflejan que, con frecuencia, no se explicita de forma adecuada y suficiente el sentido de conceptualizaciones tales como "comunicación para el cambio social» (Marí, 2016b; 2019). Así, esta etiqueta llega a aplicarse a iniciativas y proyectos que poco o nada alteran el dominio capitalista o, parafraseando a Rafael Roncagliolo (1998), a propuestas que presentan una "alternatividad poco alterativa», esto es, con escasa capacidad de alterar el orden dominante.

La comunicación para el cambio social, aquí y aho$r a$, toma cuerpo en un contexto de dependencia y marginalidad dentro del denominado capitalismo académico (Jessop, 2017). El sometimiento a la lógica de la mercancía del conocimiento, de los planes de estudios y de la gobernanza de las instituciones universitarias - por citar solamente algunas de las múltiples instancias posibles- desplaza a la periferia o, directamente al silencio, a los saberes y conoci- mientos orientados por la lógica de la emancipación social (Sousa Santos, 2009). Tomar consciencia de esta situación es un paso ineludible para construir alternativas y salir de este círculo vicioso.

El segundo frente de dependencia que se va a analizar, estrechamente conectado con el anterior, remite a la primacía del inglés en la investigación contemporánea (Ortiz, 2009). Para ello se retoman los planteamientos de Reyes Mate que afirmaba que «el pensar en español tiene que enfrentarse a un tópico más extendido de lo que parece según el cual el español no sería una lengua filosófica o, dicho de otra manera, que lenguas filosóficas son el griego y el alemán» (Mate 2008: 981). Mate explicaba esta idea con más detalle, señalando que:

"Cuando los filósofos modernos dicen y repiten que filosofía occidental es una tautología se están refiriendo a la Europa central y protestante, es decir, no a la Europa latina. ¿No decía por ventura Hegel, en su introducción a la Filosofía de la Historia, que el Weltgeist, el Espíritu Universal, la punta de lanza del desarrollo de la conciencia de la humanidad, era europeo, es decir, añadía "germánico y protestante"? El Weltgeist es centroeuropeo, germánico y protestante. España, como bien se sabe, no tocaba el manto de ese Espíritu ni de lejos pues, para él, Europa acababa en los Pirineos» (Mate, 2008: 983).

Como sucede con el pensar filosófico en español, que ha ocupado, en el imaginario y en las prácticas hegemónicas, un lugar marginal, el pensamiento comunicacional en español está alejado de la centralidad del campo a escala mundial. Según Rainer Enrique Hamel, uno de los fenómenos que influye en la creciente difusión del inglés en las ciencias es que el mundo académico anglosajón está abandonando el modelo plurilingüe tradicional a favor de un monolingüismo total y explícito, desde su posición de poder absoluto del monopolio del inglés. Estados Unidos y Reino Unido producen juntos el $41 \%$ de las publicaciones científicas (artículos en revistas) del mundo, pero el total de la difusión científica en inglés alcanza, según algunas estadísticas, más del $74 \%$ o, según otras, entre el $82 \%$ para las ciencias sociales y humanas y el $90 \%$ para las ciencias naturales (Hamel, 2005). Este proceso de concentración, además, se ha agudizado en los últimos años hasta el punto de que, en 2012, en las revistas indexadas, el español estaba presente únicamente en un $0,5 \%$ de los artículos en ciencias naturales $y$ en un $2,5 \%$ en las ciencias sociales y humanas 
(Hamel, 2013: 323). Los mismos porcentajes desproporcionados se pueden observar en el campo de las ciencias de la Comunicación. Según datos de la International Communication Association (ICA) ${ }^{3}$, la mayoría de las personas que publican en las revistas editadas por la asociación están afiliadas a universidades estadounidenses ${ }^{4}$.

Pero ¿qué consecuencias tiene esta cuestión para la investigación en comunicación? La primacía del inglés conforma "una academia altamente jerarquizada, con una hegemonía de las teorías, metodologías y estilos de pensamiento identificados con las culturas académicas particulares de los países del Atlántico Norte» (Waisbord, 2019: 94). Al profundizar en otros aspectos cualitativos igualmente trascendentes que emergen de este predominio del inglés, podemos ver que hay una presencia discreta, casi nula, de los textos de carácter teórico y que predomina la investigación empírica, en concreto, el análisis de los contenidos informativos, mientras existe una relativa desatención de las dimensiones institucional y social del sistema comunicativo (Martínez Nicolás, 2016, 2019) $)^{5}$. Es decir, se está descuidando el estudio de los aspectos vinculados con la Economía Política de la Comunicación, con el análisis de la estructura de la propiedad mediática y con las cuestiones de carácter ideológico y sociopolítico.

La práctica desaparición de la investigación teórica viene acompañada también de otros ecocidios en el plano formal. En primer lugar, el menosprecio hacia el ensayo como forma expresiva histórica y propia del campo comunicacional. La inmensa mayoría de las revistas del campo mejor indexadas $^{6}$ imponen como corsé obligatorio el estrecho esquema de la investigación hipotético-deductiva, con la obligación de realizar una producción empírica de datos, expresados en unos apartados que deben ser ordenados según la cansina secuencia repetida monotemáticamente hasta el hartazgo: objetivos-hipótesis-marco teórico-metodología-da- tos-análisis-conclusiones. Pero, como señalaba Theodor Adorno con relación a El ensayo como for$m a$, esta situación no es reciente:

"Que el ensayo en Alemania está desacreditado como producto mestizo; que carece de una tradición formal convincente; que sólo intermitentemente se ha satisfecho sus enfáticas demandas: todo eso se ha constatado y censurado bastante a menudo. La forma del ensayo sigue hasta ahora sin haber todavía cubierto el camino de autonomización que su hermana, la poesía, hace ya tiempo ha recorrido: el de la evolución a partir de una unidad primitiva e indiferenciada con la ciencia, la moral y el arte» (Adorno, 2003: 11).

Para Adorno, una de las virtudes del ensayo es que «apunta al esfuerzo del sujeto por penetrar lo que como objetividad se oculta tras la fachada» (Adorno, 2003: 12).

Otra de las restricciones impuestas por la primacía del inglés es el rechazo al dispositivo formal, propio del pensamiento latino: el uso de las metáforas, el humor, el doble sentido, la apelación a las inferencias y metarreglas comunicativas del receptor competente (Abril, 1997; Marí y Martins, 2021), que dan riqueza y alegría a la escritura, alejándola de la sosería en la que suele moverse la escritura plana y descriptiva a la que fuerzan las revistas dominantes y el sistema en general.

Sin embargo, cabe señalar, para evitar dualismos simplistas que, por fortuna, no todas las revistas anglosajonas indexadas utilizan estos esquemas, de modo que es posible encontrar en algunas de ellas espacios para los ensayos, para los textos de corte teórico y para la admisión de trabajos que aprovechan la riqueza del español. Y, a la inversa, existen también publicaciones en castellano más papistas que el Papa, que aplican, con la misma o mayor intensidad, las normas dominantes sin realizar una mínima política científica en torno al español que permita defenderlo respecto a otras formas de pensar y de expresar las ideas.

3 La ICA es una de las dos asociaciones científicas de investigación en Comunicación más relevantes del mundo junto con la International Association for Mass and Communication Research (IAMCR).

4 Este hecho explica que el 70,5\% de los artículos publicados en Journal of Communication (1956-2017), el 89,6 \% de los que se publican en Human Communication Research (1981-2017), el 79,5 \% de los aparecidos en Communication Theory (1994-2017), el 63,1\% en Journal of Computer-Mediated Communication y el 69,5\% en Communication, Culture \& Critique (2013-2017) estén escritos por personas afiliadas a universidades norteamericanas (Waisbord, 2019: 96).

5 Por ejemplo, Martínez Nicolás, en sus referenciales trabajos sobre la institucionalización de los estudios de la Comunicación en España, ha mostrado que los artículos sobre comunicación, de carácter teórico, publicados entre 1999 y 2014, constituyen el 2,4\% del total, mientras que los textos de carácter empírico publicados en el mismo periodo alcanzan casi el 80\% (Martínez Nicolás, 2019: 56).

6 En el campo de la comunicación se considera que el grupo de las revistas mejor indexadas es el compuesto por las que ocupan los dos primeros cuartiles en Scimago y Journal Citation Reports (JCR). 


\section{ADIÓS A ARISTÓTELES: LA COMUNICACIÓN NO PUEDE SER INMORAL.}

En el artículo que inspira este ensayo, Reyes Mate analizaba la disputa entre Bartolomé de las Casas y Ginés de Sepúlveda sobre la controversia en torno a la consideración de quienes habitaban en las tierras americanas bajo dominio español como sujetos de derechos. De las Casas tenía ante sí un dilema de difícil resolución: ser fiel a su formación intelectual -que tenía en Aristóteles una pieza clave- o ser fiel a la experiencia directa de injusticia y sufrimiento causada por la colonización española de América, de la que había sido testigo directo. De seguir la segunda opción entraría en contradicción con el paisaje intelectual del que formaba parte; además, como dominico, al «mandar a paseo a Aristóteles» se enfrentaría al glorioso pasado de la orden de Domingo de Guzmán, que tenía al también dominico Tomas de Aquino como una de las figuras claves en la cristianización del filósofo griego.

Años después, Mate vuelve a abordar este tema en Estos, ¿no son hombres? La pregunta en tiempos de peligro, con el fin de poner en valor el "gesto intelectual» de Bartolomé de Las Casas. La pregunta de Montesinos, que da nombre al ensayo,

«...introdujo la duda sobre la legitimidad de la conquista, y esas eran palabras mayores. Las vemos rebrotar, cuarenta años después, en la Controversia de Valladolid de 1550. Carlos V toma la sorprendente y osada decisión - única en la historia de los pueblos - de someter a debate público los títulos de la conquista. Los protagonistas son Ginés de Sepúlveda y Bartolomé de las Casas, dos primeras espadas: un moderno, Sepúlveda, que defiende la conquista, y un teólogo de formación medieval, pero al tanto de la Escuela de Salamanca, que está en su contra [...]. Su contrincante, Ginés de Sepúlveda, cuenta con notables cómplices académicos -empezando por Aristóteles, la autoridad indiscutible- que no solo distinguen entre seres inferiores y superiores, sino que asocian la inferioridad natural con la inhumanidad. No les cuesta considerar a los indígenas como seres inferiores que no poseen ninguna ciencia, que no conocen la escritura, que practican el canibalismo y hasta los sacrificios humanos [..]» (Mate, 2012: 34).

Como señala Reyes Mate, el "gesto intelectual» de Bartolomé de Las Casas le llevó a reconocer que lo primero es la experiencia de la injusticia y que si los saberes establecidos proponen interpretaciones de los hechos que agravan la injusticia, «habrá que mandar a Aristóteles a paseo»; porque «la verdad no puede ser injusta, más bien lo contrario, tiene que hacernos libres» (Mate, 2012: 34). Este hilo argumental puede completarse con algunas de las ideas desarrolladas por Reyes Mate en su Tratado de la injusticia (2011a). La obra tiene un alto poder disruptivo, en la medida en que cuestiona el lugar común de la filosofía a la hora de pensar sobre la justicia. ¿Por qué iniciar, ahora, la reflexión desde la injusticia y no desde la justicia? Para Mate, la injusticia es primera tanto histórica como lógicamente (Mate, 2012: 26) y, desde ahí, critica a la amnesia del pensamiento occidental frente al sufrimiento:

«En la medida en que el saber occidental se ha forjado sobre un yunque que no daba importancia teórica al sufrimiento - sea porque no le interesaba, sea porque no era capaz - nosotros, los nacidos después de Auschwitz, estamos obligados a tomar el sufrimiento como lo que da de pensar» (Mate, 2012: 43).

La amnesia occidental apuntada tiene que ser sustituida por una razón anamnética construida desde la memoria del sufrimiento de las víctimas. En este sentido, José Antonio Zamora (1997) ha señalado que la anamnesis «no se reduce nunca al mero conocimiento del sufrimiento pasado, sino que es, antes que eso $y$, sobre todo, un perseverante escuchar el grito de las víctimas, que se deja oír a lo largo de la historia y en el presente y que continúa exigiendo una respuesta. La razón anamnética es una razón ética precisamente por tratarse de una razón auditiva, una razón que escucha el grito de las víctimas y se deja interrumpir por él» (Zamora, 1997: 117).

Al hilo del análisis de Reyes Mate se puede plantear la siguiente pregunta: ¿cómo se hace presente Aristóteles en el campo comunicacional? Las posibles respuestas apuntan en un doble sentido:

- De un modo directo y explícito, el modelo de la retórica de Aristóteles sirve de inspiración para una de las primeras grandes teorías de la comunicación de masas elaboradas en los inicios de esta disciplina, en el primer tercio del siglo XX. El modelo de Harold Lasswell (1948), reformula en sus cinco preguntas las interrogaciones planteadas al acto comunicativo por Aristóteles (Alsina, 1995; McQuail, 2000).

- En un sentido más general, el objetivo central de la retórica aristotélica - la persuasión- es también el telos que orienta al modelo de comunicación de Lasswell, dirigido a regular el comportamiento social de las masas para que éste no entre en colisión con los intereses del poder político, económi- 
co y militar. La propuesta de Lasswell, politólogo reconvertido en lo que hoy denominaríamos un asesor en comunicación de Franklin D. Roosevelt, es una pieza clave dentro de la sociología funcionalista de los medios de comunicación, también denominada en la literatura académica como Mass Communication Research (MCR), gestada desde el poder para evitar cualquier proceso de transformación de la sociedad que altere las estructuras básicas de funcionamiento del capitalismo y de la democracia liberal. En palabras de Mattelart (1997), desde la perspectiva de Lasswell y de la MCR, el cambio social es considerado una disfuncionalidad.

Esta propuesta teórica de corte funcionalista forma parte del núcleo duro teórico que empezó a impartirse en las primeras escuelas de Periodismo y Facultades de Comunicación de Estados Unidos y de Europa a partir de la segunda mitad del siglo XX. Forma parte, por lo tanto, del bagaje ideológico y teórico que se exporta a otras regiones del mundo a la hora de configurar sus espacios universitarios y profesionales de formación en comunicación. Un ejemplo claro es el caso latinoamericano, en el que la institucionalización de los estudios en comunicación se produjo como resultado de un proceso escalonado que se prologó a lo largo de las décadas de 1940, 1950 y 1960. A partir de esta última década, en pleno proceso de gestación del movimiento crítico de los dependentistas (Katz, 2018), surgió la derivada comunicacional de esta crítica, orientada a tomar distancia de la dependencia respecto a las teorías comunicativas gestadas en Estados Unidos y a sus intereses geopolíticos subyacentes (Marí, 2019; Servaes, 2002). Este movimiento crítico conectaba, a su vez, con las demandas del Nuevo Orden Mundial de la Información y de la Comunicación (NOMIC) que, en el seno de la UNESCO, terminarán por dar lugar a uno de los mayores hitos en la formulación e institucionalización de una mirada alternativa a la comunicación: el Informe McBride (1980) (Quirós, 2013).

Frente al modelo de Lasswell y a la Mass Communication Research comenzó a articularse, con distintos epicentros en el espacio latinoamericano, una comunicación de signo alternativo, pasándose de unas teorías comunicativas al servicio del status quo, a unas teorías orientadas a la transformación estructural de la sociedad y su necesaria democratización. Los mo- delos comunicativos jerárquicos dieron paso a unos modelos de corte horizontal, participativo, popular y dialógico (Navarro y Rodríguez, 2018). El proyecto de imperialismo cultural (Schiller, 1969) dio paso a la formulación de políticas de comunicación nacionales que permitieron reposicionar a la comunicación en sus cruces con la cultura local, la ciudadanía y el desarrollo endógeno (Bustamante y Corredor, 2016).

Fueron muchas las figuras que se dedicaban a la comunicología y que destacaron por su papel en este proceso que fue dando forma al pensamiento comunicacional latinoamericano: Rosa María Alfaro, Luis Ramiro Beltrán, Juan Díaz Bordenave, Mario Kaplún, Fernando Reyes Matta, Armand Matellart, Antonio Pasquali, Eliseo Verón y un largo etcétera. Aunque cada cual aportó matices específicos, confluían en la ruptura frente a la colonización comunicacional que llegaba del Norte (preferentemente de Estados Unidos). Entre ellos, el boliviano Luis Ramiro Beltrán destaca por su conexión con el hilo conductor de este ensayo.

Beltrán nació en la ciudad de Oruro en 1930 y, desde la temprana edad de los doce años, se inició en las labores periodísticas. Su actividad profesional se desarrolló también en el campo cinematográfico, en el que destaca su guion Vuelve, Sebastiana (1955), donde situaba como narradora a una niña indígena chipaya, que da nombre a la obra, para realizar una aproximación etnográfica a su comunidad (Chaparro, 2013). Luis Ramiro Beltrán realizó sus estudios doctorales en la década de 1960 en la Universidad de Michigan. Allí conoció a impulsores de la comunicación para el desarrollo desde la perspectiva de la modernización como David Berlo, David Lerner y Everett Rogers. Como resultado del diálogo crítico que estableció con ellos, realizó un proceso de revisión crítica de sus maestros con relación a los límites de sus teorías para resolver los grandes problemas de desarrollo en Latinoamérica (Dervin, 1998: 78) ${ }^{7}$. David Berlo reconoció que Beltrán, junto a Wilbur Schramm y Paulo Freire, había provocado un cambio profundo en su modo de pensar la comunicación (Bordenave, 1998: 114). No será el único. Una de las grandes representantes de la investigación en comunicación en los Estados Unidos, Brenda Dervin- que fue la primera mujer en dirigir la prestigiosa International Communication Associationtambién habló de la profunda huella que dejó en ella

7 Previamente, en 1954, Beltrán había formado parte de un grupo latinoamericano de información agrícola formado por veintidós trabajadores. Durante tres meses, visitaron distintos centros universitarios de Estados Unidos especializados en desarrollo agrario. El paraguayo Juan Díaz Bordenave, experto como Beltrán en el campo de la comunicación y el desarrollo, formó parte de este grupo. Durante una visita que Bordenave hizo a su amigo en la Universidad de Michigan, pudo comprobar el respeto que sus compañeros y profesores le tenían (Aguirre, 2017:147). 
el pensamiento comunicacional de Beltrán en el periodo en que ambos fueron estudiantes de doctorado en Michigan. Más aún, desde su posición de peso en el campo, afirmó: «creo que ninguna otra persona ha tenido más influencia que Beltrán sobre cómo se piensa en el campo de la investigación en comunicación en Estados Unidos, respecto del quehacer académico de América Latina en materia de comunicación» (Dervin, 1998: 77). El estudio realizado en 1990 por Steven Chafee, Carlos Gómez-Palacio y Everett Rogers (1990), ya lo había indicado con anterioridad, al señalar que Luis Ramiro Beltrán es el investigador latinoamericano más citado por quienes estudian cuestiones de comunicación en Estados Unidos, sobre todo las relacionadas con América Latina ${ }^{8}$.

Dentro de la amplitud y riqueza del pensamiento comunicacional de Luis Ramiro Beltrán, destaca su obra Adiós a Aristóteles: la comunicación horizontal (1980), por las estrechas conexiones que guarda con el tema de que se ocupa este artículo. De entrada, es posible aplicar a este texto los dos distanciamientos apuntados anteriormente con relación a la MCR: respecto al esquema persuasivo de la retórica de base aristotélica y con relación al funcionalismo implícito en el enfoque modernizador de la comunicación y del desarrollo estadounidense. Pero hay un distanciamiento más sutil que permite, salvando las distancias, establecer puentes entre el mandar a paseo a Aristóteles de Bartolomé de las Casas y el Adiós a Aristóteles suyo, más educado pero situado en la misma línea. El pensador boliviano, de un modo indicial ${ }^{9}$, orientó su crítica hacia la piedra angular sobre la que se sostiene el pensamiento comunicacional hegemónico y, más aún, el pensamiento occidental dominante. Son numerosos los argumentos que, en su texto, apuntaban hacia la necesidad de impulsar modelos de comunicación horizontales, participativos y dialógicos, que pensasen de otro modo las relaciones entre comunicación, democracia y justicia social, para que se pudiesen articular otras Políticas Nacionales de Comunicación.

A pesar de ello, en su Adiós a Aristóteles, Beltrán no llegó a explicitar esta idea en toda su potencialidad. Creemos que, conscientemente, prefirió dejar parte de la crítica en el terreno de la sugerencia. Optó, así, por generar una onda expansiva como la creada por una piedra lanzada al estanque, de modo que, el dinamismo crítico que iniciaba con el sentido metafórico del título (Adiós a Aristóteles) pudiera suscitar, en quienes lo leyeran o investigaran posteriormente, procesos similares de crítica a los modelos dominantes de comunicación, de desarrollo y de sociedad. Un Adiós a Aristóteles que, en la estela de Charles S. Peirce, es indicial y simbólico ${ }^{10}$ a la vez; apuntó, en referencia directa, a Aristóteles, pero al tiempo dejó abierto un espacio a los múltiples rastros de lo aristotélico que perviven y pueden encontrarse en la cultura occidental.

\section{PENSAR LA COMUNICACIÓN DESDE EL SUR: POR UN PENSAMIENTO COMUNICACIONAL CRÍ- TICO DE IDA Y VUELTA.}

Este epígrafe presenta una reflexión sobre algunos de los debates terminológicos actuales en el campo de la comunicación para el cambio social, utilizando, de nuevo, el texto de Reyes Mate que ha servido hasta este momento como referencia. En él planteaba pensar en español de modo que «se superen tanto el universalismo abstracto que ha caracterizado a la universalidad occidental, como el casticismo o, más finamente dicho, el relativismo multiculturalista que le amenaza ahora» (Mate, 2008: 984). Para ello proponía impulsar un pensamiento desde el Sur que tuviera en cuenta las palabras de José Saramago en el Primer Congreso Iberoamericano (Cáceres-Madrid, 1998):

8 El título del trabajo de estos autores, Mass communication research in Latin America: Views from here and there, ha sido retomado y parafraseado hace unos años por Florencia Enghel y Martín Becerra (2018) quienes proponen una reflexión sobre las aportaciones del pensamiento comunicacional latinoamericano en el panorama internacional, en una línea complementaria, en parte, a la aquí expuesta.

9 El término indicial en la investigación en comunicación deriva del concepto «índice» de Charles Peirce (ver más adelante, nota 10).

10 Peirce (1893-1903), experto en semiótica y pragmática, en su texto El icono, el índice y el símbolo, aclaró, en varias ocasiones, el significado de los términos «índice» y «símbolo». Explicaba el concepto «índice» de la siguiente manera: «Un barómetro bajo con un aire húmedo es un índice de lluvia. Esto es, suponemos que las fuerzas de la naturaleza establecen una conexión probable entre el barómetro bajo con aire húmedo y la lluvia que viene. Una veleta es un índice de la dirección del viento, porque en primer lugar toma realmente la misma dirección que el viento, de modo que hay una conexión real entre ellos y, en segundo lugar, están constituidos de tal modo que, cuando vemos una veleta señalando en una cierta dirección, nuestra atención se centra en esa dirección», "el índice está conectado físicamente con su objeto». En relación con el segundo de los conceptos, señalaba: «el símbolo se conecta con su objeto en virtud de la idea de la mente que usa símbolos, sin la que no existiría ninguna conexión»; "cualquier palabra ordinaria como "dar", "pájaro", "matrimonio" es un ejemplo de símbolo. Es aplicable a cualquier cosa que se encuentre que realiza la idea conectada con la palabra. En sí misma, no identifica a esas cosas. No nos muestra un pájaro, ni realiza delante de nosotros una donación o un matrimonio, pero supone que somos capaces de imaginar esas cosas y que hemos asociado la palabra con ellas». Disponible en: https://www.unav.es/gep/IconolndiceSimbolo.html 
"Un político catalán, escribiendo sobre "La Balsa de piedra", sugirió que mi pensamiento íntimo no habría sido separar la Península Ibérica de Europa, sino transformarla en un remolque que llevase a Europa hacia el sur, apartándola de las obsesiones triunfalistas del norte y tronando solidaria con los pueblos explotados del Tercer Mundo. Es bonita la idea, pero en verdad no me atrevería a pedir tanto. A mí me bastaría con que España y Portugal, sin dejar de ser Europa, descubrieran en sí, finalmente, esa vocación de Sur que llevan reprimida, tal vez como consecuencia de un remordimiento histórico que ningún juego de palabras podrá borrar, y solo acciones positivas contribuirán a hacerlo soportable» (Saramago, 1998: 51).

Más adelante, abundando en esta idea, planteaba Mate:

«España y Portugal ya hicieron un viaje al Sur pero como norteños, es decir, conquistando y dominando. El nuevo viaje hay que hacerlo de otra manera: con conciencia de que hay un norte y un sur, países dominantes y otros dominados; y que el sur, metáfora de lo dominado por norteños del Norte y del Sur, es el punto de vista de un pensar y de un actuar creativo. Y lo serán en la medida en que pensamiento y acción construyan un futuro mirando hacia atrás, es decir, entiendan que la construcción de un mundo nuevo que no sea reproducción de lo que de siempre dependerán de cómo interpreten su responsabilidad con el pasado» (Mate, 2008: 985-986).

En los últimos años, en esta misma línea, Boaventura de Sousa Santos (2009) ha planteado en varias de sus obras una sugerente reflexión en torno a las epistemologías del Sur que históricamente han sido arrinconadas y silenciadas por el pensamiento dominante del Norte y sus criterios tradicionales de demarcación de las ciencias. En Justicia entre Saberes (2017) se preguntaba si hay espacio para pensar en un «Occidente no occidentalista», refiriéndose con esta expresión «a una vasta diversidad de concepciones, teorías y argumentos que, aunque eran producto de reconocidas figuras intelectuales occidentales, fueron descartados, marginados e ignorados porque no encajaban en los objetivos políticos del capitalismo y el colonialismo que sirven de fundamento para la construcción de la singularidad y superioridad de la modernidad occidental»(Sousa Santos, 2017: 137). Rescatando ideas de pensadores como Luciano de Samosata, Nicolás de Cusa y Blaise Pascal, quería «ejemplificar algunos de los caminos que nos pueden llevar a la construcción de un diálogo intercultural no capitalista y no colonialista» (Sousa Santos, 2017: 134).

A partir de las fisuras identificadas por Sousa Santos en el pensamiento occidental, se abre la posibilidad de seguir explorando otros autores y otros conceptos dignos de ser rescatados para pensar un poscapitalismo no eurocéntrico. Silvio Waisbord (2019) continúa con esta reflexión, al proponer "deswesternizing» (que puede traducirse como desoccidentalizar) los estudios en comunicación, en una línea que esbozó años antes en un trabajo previo escrito junto a Claudia Mellado (2014). Con este concepto remiten a «un cambio en el conocimiento académico para ampliar el análisis considerando las experiencias, los resultados de la investigación y los marcos teóricos desarrollados en el resto del mundo. La desoccidentalización se considera necesaria para enriquecer un campo que se ha organizado históricamente en torno a conceptos analíticos, epistemologías, argumentos y pruebas desarrollados en los Estados Unidos y Europa Occidental» (Waisbord y Mellado, 2014: 100).

El problema, por tanto, no reside en el qué (la desoccidentalización del conocimiento) sino en los cómos: los procesos y los cauces institucionales necesarios para llevar adelante esta tarea. En la actualidad, existe un movimiento creciente en el campo de la investigación y del activismo social, que defiende la necesidad de sensibilizar frente a la dimensión colonial presente en gran parte del conocimiento construido históricamente en Occidente. Sin embargo, a la hora de articular y encauzar este giro decolonial surgen diferencias. Como señala Silvio Waisbord «no está claro el modo en que los estudios de comunicación se involucrarían en la autorreflexividad y abrazarían una sensibilidad desoccidentalizada y cosmopolita; los elocuentes llamamientos a problematizar los fundamentos del conocimiento basado en Occidente son importantes, pero no son suficientes si se tiene en cuenta la estructura actual de los estudios académicos mundiales» (Waisbord, 2019: 106) ${ }^{11}$.

Así, existen varias cuestiones que requieren una reflexión más profunda, entre ellas la tendencia a un pensamiento dualista en la crítica de la colonialidad

11 El término autorreflexividad aplicado a una persona hace referencia a la reflexión sobre uno/a mismo/a. En el campo de las ciencias de la comunicación, como es el caso del uso de la palabra en la cita de Silvio Wasibord, se refiere a la reflexión que hace un campo de la investigación, en este caso de las ciencias de la comunicación, sobre sí mismo. 
y la occidentalización del conocimiento, que puede llevar a idealizar al Sur y a demonizar al Norte. En el campo de la comunicación, este dualismo tiene su correlato en la propuesta de Waisbord y Mellado (2014) y en otras formulaciones similares. Ya en la década de 1990, Robert Huesca y Brenda Dervin realizaban una interesante reflexión en torno al «legado de los dualismos» que se manifestaba en las propuestas de la comunicación alternativa, formuladas en oposición a las propuestas dominantes. Comunicación horizontal vs comunicación vertical, enfoque democrático vs. enfoque autoritario, diálogo vs. monólogo y, así, una larga lista de pares de opuestos utilizados para clasificar en dos compartimentos estancos ambos enfoques (Huesca y Dervin, 1994: 58).

Huesca y Dervin planteaban ir más allá de los dualismos mediante la incorporación de lo oposicional en el enfoque alternativo. Para ellos, la investigación crítica latinoamericana había tendido a establecer un retrato bidimensional del mundo que, si ya en las décadas de 1970 y 1980 mostraba serias limitaciones, con el paso del tiempo fueron mucho más evidentes, en un contexto de mayor complejidad. Las prácticas de comunicación popular socavaron muchos de los supuestos instrumentales que guiaban la comunicación alternativa y, al mismo tiempo, los estudios transnacionales permitieron ver que la comunicación alternativa no era impermeable a la influencia de los procesos globales (Huesca y Dervin, 1994: 65).

Los agudos análisis de Huesca y Dervin resultan de gran ayuda para identificar estos procesos dualistas en los debates actuales en torno a la relación entre la teoría crítica occidental y las propuestas alternativas procedentes del Sur Global, defendidas por algunos enfoques decoloniales en Comunicación. El retrato bidimensional ofrece una imagen simplificada, carente de profundidad, en la que no se perciben suficientes referencias a los contextos y a los procesos en los que las teorías viajan, favorecen el desarrollo de un pensa- miento crítico e impulsan procesos emancipatorios, independientemente de su partida de nacimiento. El sugerente concepto "travelling theories» de Edward Said contribuye a superar las limitaciones del pensamiento dualista, al tiempo que ilustra mejor los procesos de intercambio teórico que se llevan a cabo en nuestro mundo globalizado. Said propone la metáfora del viaje para seguir la evolución de la teoría crítica del siglo XX e identifica, en este proceso, cuatro etapas: 1) partida desde el lugar de origen; 2 ) paso a través de diferentes contextos; 3) «transplante» («transplantation», en el inglés original) de las teorías en un nuevo contexto, con sus propias condiciones de aceptación, y (4) re-emergencia de la idea inicial, transformada por el desplazamiento y por los nuevos usos (Said, 1983) ${ }^{12}$.

Durante mucho tiempo, se ha defendido que los conceptos y teorías dominantes han viajado en un único sentido - del Norte al Sur -, invisibilizándose las propuestas teóricas que, desde el Sur, llegaban al Norte. Sin embargo, en nuestros días, en el campo de la comunicación algunas teorías e imaginarios del Sur Global están siendo asumidas por quienes investigan de forma crítica sobre comunicación en el Norte, por su capacidad para generar nuevos conceptos y prácticas emancipatorias, como es el concepto quechua "Sumak Kawsay» ("Buen Vivir», en su traducción al castellano) y el sudafricano "Ubuntu», que albergan un gran potencial para articular la crítica al discurso eurocéntrico. ${ }^{13}$ Así, el desequilibrio histórico de estos flujos de ideas del Norte al Sur, está comenzando a revertirse. Además, se han identificado conceptos que viajan bien de un lado a otro y otros que no lo hacen tanto. En este sentido, Pradip Thomas (2020), investigador destacado del campo de la comunicación para el cambio social, retoma el concepto de «acumulación por desposesión ${ }^{14}$ de David Harvey (2004) para analizar la situación de las comunidades de los irulas en India, presentándolo como uno de los conceptos que viajan bien del Norte al Sur.

12 James Clifford reflexionaba sobre este mismo concepto, a partir de su propuesta de travelling cultures (Clifford, 1992). Charles Forsdick (2001) también retoma la idea de Said para reflexionar en torno a los travelling concepts.

13 El concepto quechua "Sumak Kawsay ha sido teorizado, entre otros, por Alberto Acosta (2013). Sobre el concepto de origen africano "Ubuntu», Nelson Mandela y Desmond Tutu, entre otros, han propuesto definiciones. Ver: https://www.beliefnet.com/inspiration/2004/04/desmond-tutus-recipe-for-peace.aspx

14 El término hace referencia a los procesos de acumulación del sistema capitalista que permiten su perpetuación y legitimación, tanto en el momento histórico del paso del feudalismo al capitalismo como en la actualidad. Resulta especialmente interesante conocer los procesos de los que habla Harvey mediante los cuales se llevó a cabo, en el naciente capitalismo de la Edad Media, la acumulación primitiva. Estos procesos son, entre otros: la mercantilización y privatización de la tierra, la conversión de varios tipos de derechos de propiedad (comunal, colectiva, estatal, etc.) en derechos de propiedad privada exclusiva; la supresión de acceso a los bienes comunales; la mercantilización de la fuerza del trabajo y la monetarización de los intercambios y de los impuestos, en particular sobre la tierra (Harvery, 2004:116). 
En el campo de la música existen también, ejemplos válidos de teorías viajeras. Es el caso del flamenco, que incluye entre uno de sus palos los cantes de ida y vuelta, que incorporan elementos de una serie de "cantes aflamencados de origen hispanoamericano" como la guajira, la rumba, la vidalita, la milonga o la colombiana, entre otros (Blas Vega, 1997) ${ }^{15}$. La inclusión de estos elementos del folclore del centro y del sur de América supone un enriquecimiento del cante flamenco. Estas músicas llegaron a España traídas por artistas que habían viajado a América o procedentes de la memoria popular de los aires de ultramar.

Siguiendo el leitmotiv que articula este ensayo, proponemos el término de teorías de ida y vuelta como una adaptación de la expresión anglosajona travelling theories, para dar cuenta de los procesos de mestizaje e hibridación en los que frecuentemente se ven sumergidos los intercambios de ideas. La metáfora del viaje continuo en el doble sentido permite visualizar las estrategias de apropiación que las teorías críticas establecen cuando viajan desde lo español hasta lo latinoamericano y viceversa. Así ha sucedido, históricamente, en el campo de la investigación en Comunicación, cuando se exploran los itinerarios formativos e investigadores de autores y autoras clave del pensamiento comunicacional latinoamericano. Es el caso, por ejemplo, del venezolano Antonio Pasquali, uno de los introductores de la Teoría Critica en Latinoamérica en el campo comunicacional, que realizó sus estudios doctorales en la universidad de la Sorbona (Francia). También del argentino Eliseo Verón, semiólogo de proyección internacional, que estudió en el Collège de France con Lévi-Strauss e impartió docencia en varias universidades francesas, hasta obtener el diploma de Doctor de Estado en 1985 por la Universidad Paris VIII. Sus itinerarios investigadores son dos botones de muestra del recorrido que siguieron las teorías críticas en comunicación en su viaje hasta el espacio latinoamericano. Unas ideas críticas que llegaron, en los momentos iniciales, más de Europa que de Estados Unidos.

Ya vimos anteriormente los embates teóricos que mantuvieron Luis Ramiro Beltrán y Juan Díaz Bor- denave con el funcionalismo y el empirismo comunicativo que llegaba, en la década de 1950 y 1960 desde los Estados Unidos a América Latina. Sin olvidar estas aportaciones históricas, corren tiempos en los que Europa está llamada a prestar atención a los cantes que llegan del pensamiento comunicacional latinoamericano, con el fin de fecundar y revitalizar las teorías críticas en el campo de la comunicación, también, en sus flujos del Sur al Norte.

\section{CONCLUSIÓN: MÁS ALLÁ DE LA TEORÍA CRÍTICA DERIVATIVA Y DEL PENSAMIENTO POSTADÁNICO}

Como se ha visto a lo largo de este ensayo, las aportaciones de la comunicación para el cambio social, siguiendo a Lluís Duch (2004), están imbuidas de la «condición adverbial» que caracteriza a toda actividad humana. De ahí que se haya utilizado el título original de Reyes Mate para este ensayo, pues se ha considerado que las múltiples transiciones en las que está inmerso nuestro objeto de estudio hacen que las reflexiones sobre el mismo también estén en movimiento.

La comunicación para el cambio social en español está situada entre el polo de tensiones que oscila desde los límites de la teoría crítica derivativa a las propuestas de reformulación inmersas en las lógicas del «lenguaje postadánico» ${ }^{16}$. Siguiendo a Sousa Santos (2009), en el primer polo de la tensión, es posible constatar que el pensamiento crítico se ha vuelto derivativo, esto es, que el uso de los sustantivos viene marcado por el pensamiento hegemónico, quedando al pensamiento crítico, únicamente, espacio para los adjetivos, con lo que se «acepta la necesidad de enmarcar sus debates y propuestas en un horizonte de posibilidades que inicialmente no es el suyo" (Sousa Santos, 2017: 96). En el extremo opuesto, la urgencia por salir de este enredo, más a fuerza de voluntad que de análisis, puede llevar a unas lógicas propias de lo que Benjamin denominaba "lenguaje postadánico», un lenguaje que «suple su incapacidad de nombrar con aproximaciones lingüísticas que rondan la charlatanería» (Mate, 2012: 71).

15 Ana Vega-Toscano, en su artículo «Los cantes de ida y vuelta en el flamenco: la guajira», junto a la expresión "cantes de ida y vuelta», planteaba que José Blas Vega, entre otras personas expertas, han propuesto el término «cantes aflamencados de origen latinoamericano» (Vega-Toscano, 1997: 973).

16 Reyes Mate (2012) recupera elementos de la teoría benjaminiana del lenguaje, en la que se distingue entre el lenguaje adánico y el postadánico. El primero tiene la capacidad de nombrar las cosas en conexión con su esencia lingüística. Por otro lado, el lenguaje postadánico hace referencia al periodo posterior a la salida del paraíso del ser humano y, según Mate, "suple su incapacidad de nombrar con aproximaciones linguísticas que rondan la charlatanería» (Mate, 2011b: 71). Walter Benjamin explicó los conceptos «adánico» y "postadánico» en On Language as Such on the Language of Man (Benjamin, 1916). 
Así, la comunicación para el cambio social en español tiene ante sí la necesidad de superar el peligro de una proliferación de términos alternativos a los dominantes que, más allá del ruido generado por la provocación formal, no lleguen a ser alterativos del desorden y de las injusticias sociales generadas. La ruptura de la dependencia respecto a nuestra Scila -las lógicas de dominio del capitalismo global colonizador- puede conducir, sin pretenderlo, a chocar con nuestra Caribdis, esto es, la propagación de una polifonía de términos alternativos que devenga en una cacofonía generadora de un ruido incapaz de movilizar el pensamiento y la acción emancipatorios.

Las vías de salida de esta comunicación alterativa recuerdan al dilema moral y ético al que se enfrentó Bartolomé de las Casas en el siglo XVI, que nos ha enseñado que los nuevos términos y argumentaciones que emanen de la comunicación para el cambio social no pueden perder de vista la inmoralidad de la injusticia social imperante. Y, por lo tanto, esta, la inmoralidad de la injusticia social, será una brújula fundamental para orientar los caminos futuros de reformulación teórica del pensamiento comunicacional crítico de aquí y de allá.

\section{AGRADECIMIENTOS:}

Este artículo forma parte del proyecto de investigación de la AEI (Agencia Estatal de Investigación) "Comunicación Solidaria Digital. Análisis de los imaginarios, los discursos y las prácticas comunicativas de las ONGD en el horizonte de la Agenda 2030" (PID2019106632GB-I00/AEI/10.13039/501100011033) IP: Víctor Manuel Marí Sáez. Es resultado, también, de la estancia de investigación financiada por el Plan Propio de la Universidad de Cádiz (EST2021-051), que el autor ha realizado en el Centro de Ciencias Humanas y Sociales (CCHS) del Consejo Superior de Investigaciones Científicas (CSIC) gracias a la invitación del Prof. Dr. José Antonio Zamora Zaragoza.

\section{REFERENCIAS}

Acosta, Alberto (2013). El Buen Vivir. Sumak Kawsay. Una oportunidad para imaginar otros mundos. Barcelona: Icaria.

Aguirre, José Luis (2017). Diálogo entre entrañables comunicadores: Juan Díaz Bordenave y Luis Ramiro Beltrán Salmón. Revista Latinoamericana de Ciencias de la Comunicación, 13 (25): 145-154.

Abril, Gonzalo (1997). Teoría General de la Información. Madrid: Cátedra.

Adorno, Theodor (2003). Notas sobre la literatura. Obra completa (11). Madrid: Akal. [Traducción del original publicado por Suhrkamp Verlag, Frankfurt am Main, 1974].

Beltrán, Luis Ramiro (1980). A Farewell to Aristotle: horizontal communication. Communication, 5: 5-41.

Benjamin, Walter (1916). On Language as Such on the Language of Man. En Marcus Bullock y Michael W. Jennings (1996). Walter Benjamin. Selected Writings (Vol. 1), 1913-1926 (pp. 245-260). Cambridge, MA: Harvard University Press.

Bustamante, Enrique y Corredor, Patricia (2016). Política de comunicación y la cultura en España. Balance de una arti- culación siempre precaria. En Joaquim Rius y Juan Antonio Rubio (coords.). Treinta años de políticas culturales en España (pp. 245-260). Valencia: Universitat de Valencia.

Chaffee, Steven; Gómez-Palacio, Carlos y Rogers, Everett. (1990). Mass communication research in Latin America: Views from here and there. Journalism Quarterly, 67 (4): 1015-1024. https://doi.org $/ 10.1177 / 107769909006700402$

Chaparro, Manuel (2012). Medios de comunicación y democracia. Lecciones desde América Latina. Commons. Revista de Comunicación y Ciudadanía Digital, 1 (1): 15-34. https://dx.doi.org/10.25267/ COMMONS.2012.v1.i1.03

Chaparro, Manuel (2013): Memorias chipayas. Madrid:Fragua.

Clifford, James (1994). Traveling cultures. In Lawrence Grossberg, Cary Nelson y Paula A. Treicher (eds.). Cultural Studies (pp. 96-112). London: Routledge.

Couldry, Nick y Hepp, Andreas (2013). Conceptualizing Mediatization: Contexts, Traditions, Arguments. Communication Theory, 23 (3): 191-202. https://doi. org/10.1111/comt.12019

Dervin, Brenda (1998). En alguna parte entre la poesía y la prosa, el hecho y el sentimiento, las superficies y los secretos, Luis Ramiro Beltrán, el campo de la comunicación en EE. UU. y Yo. En José Marques de Melo y Juçara Brittes (eds.) (1998). A trajetória comunicacional de Luis Ramiro Beltrán (pp. 178-189). Sao Bernardo do Campo: Universidade Metodista de Sao Paulo.

Duch, Lluis (2004). Estaciones del laberinto. Ensayos de antropología. Barcelona: Herder

Enghel, Florencia y Becerra, Martín (2018). Here and There: (Re)Situating Latin America in International Communication Theory. Communication Theory, 28 (2): 111-130. https://doi.org/10.1093/ ct/qty005

Forsdick, Charles (2001). Travelling Concepts: Postcolonial Approaches to Exoticism. Paragraph, 43 (2): 12-29. https:// doi.org/10.3366/jsp.2001.24.3.12

Fuchs, Christian (2020). Communication and Capitalism: A Critical Theory. London: University of Westminster Press. https://doi.org/10.2307/j.ctv12fw7t5

Golombek, Diego (comp.) (2006). Demoliendo papers. La trastienda de las publicaciones científicas. Buenos Aires: Siglo XXI.

Gumucio, Alfonso y Tufte, Thomas (eds.) (2006). Communication for Social Change Anthology: Historical and Contemporary Readings. New Jersey: Communication for Social Change Consortium. 
Hamel, Rainer Enrique (2005). El español en el campo de las ciencias: propuestas para una política del lenguaje. En Centro de Estudios Lingüísticos y Literarios (ed.). Congreso Internacional sobre Lenguas Neolatinas en la Comunicación Especializada (pp. 87-112). México: Agence Intergouvernamentale de la Francophonie. México: El Colegio de México, Unión Latina.

Hamel, Rainer Enrique (2013). El campo de las ciencias y la educación superior entre el monopolio del inglés y el plurilingüismo: elementos para una política del lenguaje en América Latina. Trabalhos em Linguística Aplicada, 52 (2): 321-384. https://doi.org/10.1590/ S0103-18132013000200008

Harvey, David (2004). El nuevo imperialismo. Madrid:Akal.

Huesca, Robert y Dervin, Brenda (1994). Theory and Practice in Latin American Alternative Communication Research. Journal of Communication, 44 (4), pp. 53-73. https://doi.org/10.1111/j.1460-2466.1994. tb00699. $x$

Jessop, Bob (2018). On academic capitalism. Critical Policy Studies, 12 (1): 104109. https://doi.org/10.1080/1946017 1.2017.1403342

Katz, Claudio (2018). La Teoría de la Dependencia, cincuenta años después. Buenos Aires: Batalla de Ideas.

Lasswell, Harold (1948). The Structure and Function of Communication in Society. The Communication of Ideas. Nueva York: Institute for Religious and Social Studies.

Marí, Víctor Manuel (2016a). Comunicaciones ininterrumpidas. Madrid: PPC.

Marí, Víctor Manuel (2016b). Communication, development, and social change in Spain: A field between institutionalization and implosion. International Communication Gazette, 78 (5): 469-486. https:// doi.org/10.1177/1748048516633616

Marí, Víctor Manuel y Ceballos, Gonzalo (2019). Opening the Black Box of Citations: A Qualitative Analysis on the Basis of the Taxonomy of Erikson and Erlandson. Communication Theory, 29 (4): 463481. https://doi.org/10.1093/ct/qty027

Marí, Víctor Manuel y Martins, Clara (2021). Communication Research, the Geopolitics of Knowledge and Publishing in
High-Impact Journals: the Chronicle of a Commodification Process Foretold. TripleC. Communication, Capitalism \& Critique 19 (2): 307-324. https://doi. org/10.31269/triplec.v19i2.1258

Marques de Melo, José y Brittes, Juçara (eds.) (1998). A trajetória comunicacional de Luis Ramiro Beltrán. Sao Bernardo do Campo, Brasil: Universidade Metodista de Sao Paulo.

Marques de Melo, José (2009). Pensamiento comunicacional latinoamericano. Entre el saber y el poder. Sevilla: Comunicación Social.

Martínez Nicolas, Manuel (2006). Masa (en situación) crítica: la investigación sobre el periodismo en España: comunidad científica e intereses de conocimiento. Análisi. Quaderns de comunicació i cultura, 33:135-170.

Martínez Nicolás, Manuel; Saperas, Enric y Carrasco-Campos, Ángel (2019). La investigación sobre comunicación en España en los últimos 25 años (19902014). Objetos de estudio y métodos aplicados en los trabajos publicados en revistas españolas especializadas. Empiria. Revista de Metodología en Ciencias Sociales, 42: 37-69. https://doi. org/10.5944/empiria.42.2019.23250

Marzal, Javier, Rodríguez, Aaron y Soler, María (2018). Los estudios de comunicación: entre las humanidades y las ciencias sociales. Una aproximación desde el Ranking de Shangai. En Rodríguez, Aaron y Gil, Samuel (eds.): Investigar en la era neoliberal. Visiones críticas sobre la investigación en Comunicación en España (pp. 109-120). Barcelona: Servicio de Publicaciones de la Universitat Autónoma de Barcelona. https://doi. org/10.7203/PUV-ALG38-313-4

Mate, Reyes (2008). Pensar en español aquí y ahora. Arbor. Ciencia, Pensamiento y Cultura. CLXXXIV: 979-988. https://doi. org/10.15332/s0120-8462.2012.0107.02

Mate, Reyes (2011a). Tratado de la injusticia. Barcelona: Anthropos.

Mate, Reyes (2011b). ¡A paseo Aristóteles!: cómo pensar en español. Claves de razón práctica, 212: 70-76.

Mate, Reyes (2012). Estos, ¿no son hombres? La pregunta en tiempos de peligro. Cuadernos de Filosofía Lationamericana, 33 (107): 29-35.
Mattelart, Armand y Mattelart, Michèle (1997). Historia de las Teorías de la Comunicación. Barcelona: Paidós.

McQuail, Denis (2000). Introducción a la teoría de la comunicación de masas. Barcelona: Paidós.

Navarro, Felipe y Rodríguez Marino, Paula (2018). Aproximaciones conceptuales: comunicación popular, comunicación comunitaria y comunicación alternativa. Commons. Revista de Comunicación y Ciudadanía Digital, 7 (2): 37-66. DOI: http://dx.doi.org/10.25267/COMMONS.2018.v7.i2.02

Ortiz, Renato (2009): La supremacía del inglés en las ciencias sociales. México: Siglo XXI.

Peirce, Charles Sanders (1893). Peirce Logic Notebook (1865-1909). Cambridge: MA, Harvard University.

Quirós, Fernando (2013). El debate sobre la información, la comunicación y el desarrollo en la UNESCO durante el siglo XX. Commons. Revista de Comunicación y Ciudadanía Digital, 2 (2): 7-38. http://dx.doi.org/10.25267/COMMONS.2013.v2.i2.01

Rodrigo Alsina, Miquel (1995). Los modelos de comunicación. Madrid: Tecnos.

Roncagliolo, Rafael (1998). Las redes de cooperación y radio comunitaria. En Chaparro, M. (coord.) La democratización de los medios (pp. 259-268.). SeviIla: Diputación de Sevilla/EMA-rtv.

Said, Edward Wadie (1982). Traveling Theory. En Edward Wadie Said (ed.). The World, the Text, and the Critic (pp. 226-247). Cambridge, MA: Harvard University Press.

Servaes, Jan (2002). Approaches to Development Communication. París: UNESCO.

Schiller, Herbert (1969). Mass Communications and American Empire. New York: Kelley.

Sousa Santos, Boaventura (2009). Una epistemología del Sur: la reinvención del conocimiento y la emancipación social. Buenos Aires: Siglo XXI. https:// doi.org/10.2307/j.ctvnp0k5d.4

Sousa Santos, Boaventura (2017). Justicia entre Saberes. Epistemologías del Sur contra el epistemicidio. Madrid: Morata. 
Thomas, Pradip (2020). The Imperialism of Categories: Concepts and Contexts in Communication for Social Change. Communication Theory, 30 (4): 388-406. https://doi.org/10.1093/ct/qtz026
Waisbord, Silvio y Mellado, Claudia (2014). De-westernizing Communication Studies: A Reassessment. Communication Theory, 24 (4): 361-372. https://doi. org/10.1111/comt.12044
Waisbord, Silvio (2019). Communication. A Post-Discipline. Cambridge: Polity Press.

Zamora, José Antonio (1997). Religión y crisis de Dios, hoy. Scripta Fulgentina: revista de Teología y Humanidades, 7 (13): 109-123. 